\title{
A wearable phototherapy device utilizing micro-LEDs
}

\author{
Francesca Farrell*, Enyuan Xie*, Benoit Guilhabert*, Anne-Marie Haughey ${ }^{\dagger}$, Patricia Connolly \\ Martin D Dawson* and Nicolas Laurand* \\ *Institute of Photonics, Dept of Physics, SUPA, University of Strathclyde, Glasgow, UK \\ ${ }^{\dagger}$ Fraunhofer Centre for Applied Photonics, Glasgow, UK \\ ‡Department of Biomedical Engineering, University of Strathclyde, Glasgow, UK \\ Email: francesca.farrell@strath.ac.uk
}

\begin{abstract}
A conformable device for wearable phototherapy applications is presented. The device consists of a $1 \mathrm{~mm}$ thick elastomeric membrane edge-lit by specially fabricated microsized LEDs. Nanoparticle based scattering films are utilized to extract light and a uniform emission of $15 \mu \mathrm{W} / \mathrm{cm}^{2}$ is reported over an area of $2 \mathrm{~cm}^{2}$.
\end{abstract}

\section{INTRODUCTION}

Wearable photonic devices have many applications in the healthcare field, including sensors for physiological monitoring and light sources for phototherapy devices [1]-[3]. Phototherapy is the treatment of skin disorders such as eczema, psoriasis and acne with UV or visible light [4], [5]. The wavelength of light utilized is dependent on the condition to be treated, as UV and blue wavelengths penetrate the epidermis whilst red and NIR wavelengths penetrate deeper into the dermis [6]. In some cases a photosensitizing drug is applied to the skin before light exposure to further enhance the effect of the light [7]. Traditionally, phototherapy and PDT are carried out in a clinical environment utilizing bulky lamp systems however, in order to improve patient convenience and decrease demand on healthcare resources, at-home devices are being developed [8], [9]. These devices consist of LED arrays that directly irradiate the skin and are worn on the body giving the patient improved comfort. The rigidity of these devices make it difficult for clinicians to determine the treatment dosage due to their limited conformability and the hot-spots in their light distribution [2], [10]. The development of flexible light technologies could be a solution to this problem; a flexible and conformable device would allow for a maximum treatment efficiency with a potentially lower power over a longer treatment time.

As a light source for phototherapy, LEDs are beneficial due to their high efficiencies, low-cost design and their miniaturization abilities. They can also be fabricated and combined in a variety of wavelengths, making them ideal for multi-wavelength emission. In order to avoid hot-spots, LEDs can be placed at the edge of a transparent substrate and light guiding/extracting features can be utilized to produce a uniform emission. To develop a truly thin device, microsized GaN LEDs ( $\mu$ LEDs) can be used; these are usually less than $100 \mu \mathrm{m}$ in size and can be fabricated into a variety of pixel shapes and sizes [11], [12]. The small size of these LEDs allows for high coupling efficiencies to sub-mm thick substrates.
Herein, we combine custom $\mu$ LEDs with a millimeter thick polydimethylsiloxane (PDMS) membrane. PDMS is a flexible, biocompatible polymer that can act as an effective waveguiding material [13]. In order to extract the guided light, $\mathrm{TiO}_{2}$ nanoparticles are embedded in PDMS substrates and added to the top surface of the light sheet to obtain an extracting region over an area of $2 \mathrm{~cm}^{2}$.

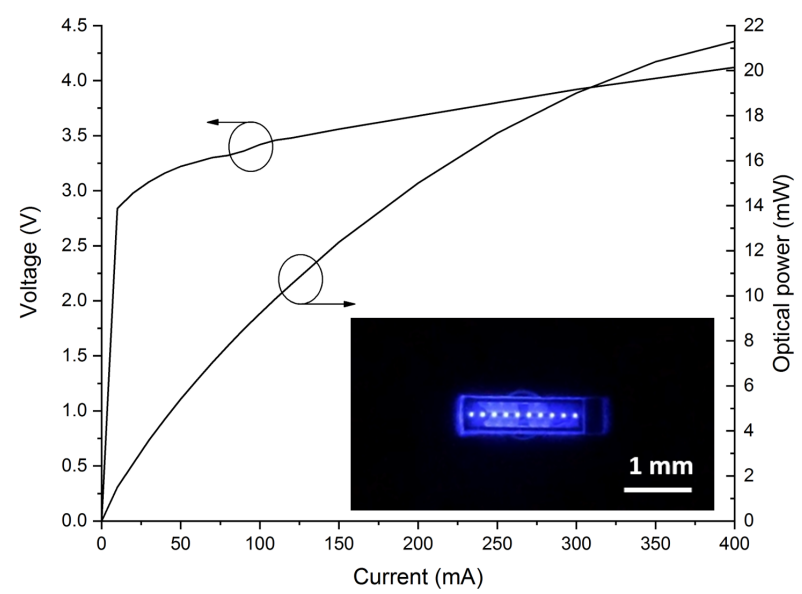

(a)

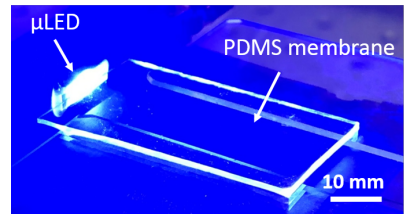

(b)

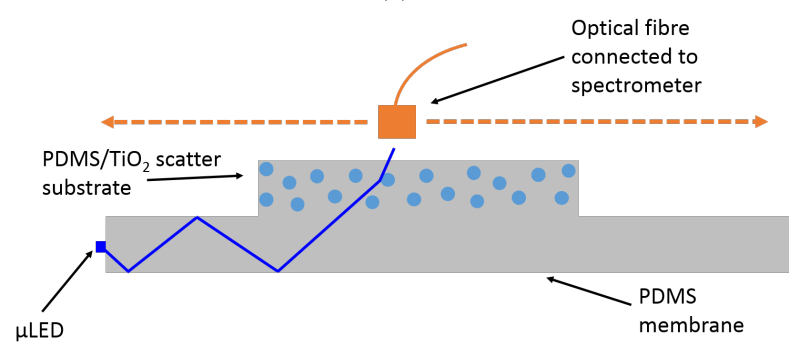

(c)

Fig. 1: The voltage-current-optical power characteristics of the $\mu$ LEDs, inset shows the 10 individual pixels, (a) $\mu$ LED array combined to a $1 \mathrm{~mm}$ thick PDMS membrane (b) and schematic of the irradiance measurement set up (c). 


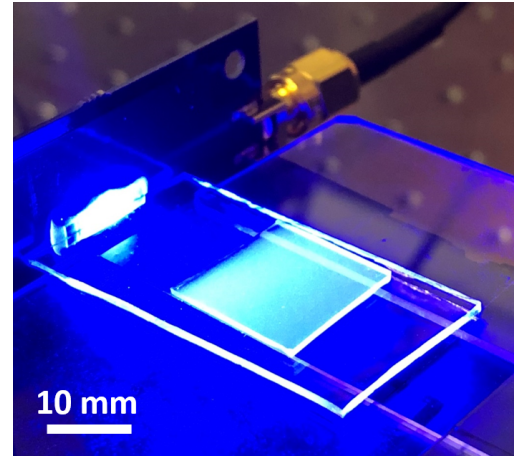

(a)

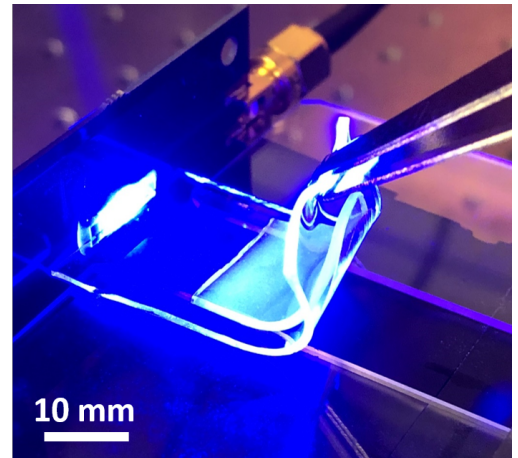

(b)

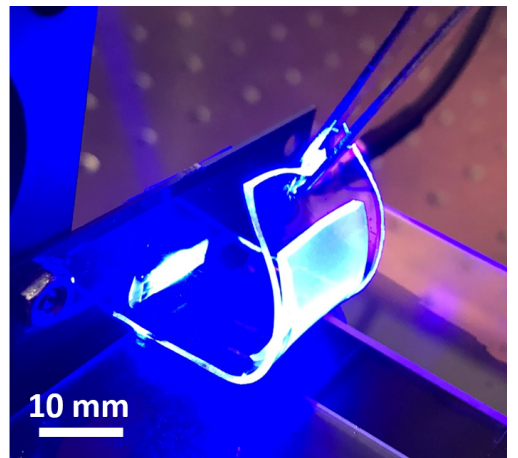

(c)

Fig. 2: Image of the $0.25 \mathrm{wt} \%$ scatter substrate on the PDMS membrane showing the extraction effect (a), images showing the flexibility of the device (b) and $(\mathrm{c})$.

\section{MATERIALS AND METHODOLOGY}

\section{A. Device design}

The light source utilized is a custom $\mu$ LED array consisting of 10 pixels, each $100 \times 100 \mu \mathrm{m}^{2}$ with a pitch of $720 \mu \mathrm{m}$, shown in Fig. 1a. The voltage-current-optical power characteristics of the LED array are given. In this study the $\mu$ LED device is driven at a current of $110 \mathrm{~mA}$, providing a total optical power of $9 \mathrm{~mW}$ with a peak emission of $444 \mathrm{~nm}$.

The PDMS membrane in this study is made from a ratio of 5:1, silicone to cross-linker (RTV615), cured at room temperature for 48 hours to produce a flexible film with dimensions $40 \times 20 \times 1 \mathrm{~mm}^{3}$. Based on the thickness of the PDMS membrane and the size of the $\mu$ LEDs, the maximum coupling efficiency of the device is estimated to be $92 \%$ [14].

\section{B. Light extraction - scattering structure}

Scattering structures are fabricated by mixing different weight ratios of $\mathrm{TiO}_{2}$ nanoparticles (mean diameter $25 \mathrm{~nm}$ ) with PDMS at different weight ratios. The resulting substrates $\left(15 \times 15 \times 1 \mathrm{~mm}^{3}\right)$ are cured at room temperature for 48 hours before being placed directly on the PDMS membrane at a distance of $15 \mathrm{~mm}$ from the LED.

\section{Uniform light extraction - tapered scattering structure}

Diffusing structure is fabricated as before, however it is cured at an angle of $15^{\circ}$ to produce a tapered substrate that increases in thickness along the length, from $0.5 \mathrm{~mm}$ to a maximum of $4 \mathrm{~mm}$. This substrate is placed on the membrane, $15 \mathrm{~mm}$ from the LED.

\section{Irradiance measurements and mapping}

An optical fiber and calibrated spectrometer are used to measure the scattered light from the top surface of the light sheet as irradiance $\left(\mu \mathrm{W} / \mathrm{cm}^{2}\right)$. The fiber is set at a height of $1 \mathrm{~mm}$ from the top surface of the light sheet and is translated along the membrane, with increasing distance from the LED, to obtain irradiance measurements along one direction as shown in Fig. 1c. To obtain a 2D map of irradiance over the extraction area, a CCD and lens system are utilized.

\section{RESULTS}

\section{A. Light extraction}

Fig. 2a shows the extraction effect of the $0.25 \mathrm{wt} \%$ $\mathrm{TiO}_{2} /$ PDMS scatter substrate on the PDMS membrane, Fig. $2 b$ and $c$ show that the flexibility of the device is not hindered by the additional layer. Fig. 3 plots the irradiance as a function of distance from the LED for the PDMS membrane alone and with a range of scatter substrates utilizing different weight $\%$ of $\mathrm{TiO}_{2}$ to PDMS. Using the PDMS as a reference it can be seen that the amount of scattered light decreases with distance from the LED, excluding the end of the membrane $(40 \mathrm{~mm})$ where there is an increase due to edge effect. A maximum irradiance of $9 \mu \mathrm{W} / \mathrm{cm}^{2}$ is obtained closest to the LED, this decays rapidly and between 15 and $35 \mathrm{~mm}$ there is only a small amount of scattered light $\left(0.4 \mu \mathrm{W} / \mathrm{cm}^{2}\right.$ average $)$ as this is where most of the light is guided. As can be seen in Fig. 2 and Fig. 3, the $\mathrm{TiO}_{2} / \mathrm{PDMS}$ substrate acts as an extractor for the light from the membrane. The amount of light extracted is dependent on the $\mathrm{TiO}_{2}$ wt $\%$.

Different weight concentrations of $0.05 \%$ to $1 \%$ were utilized. From the results it can be seen that as the $\mathrm{TiO}_{2}$ con-

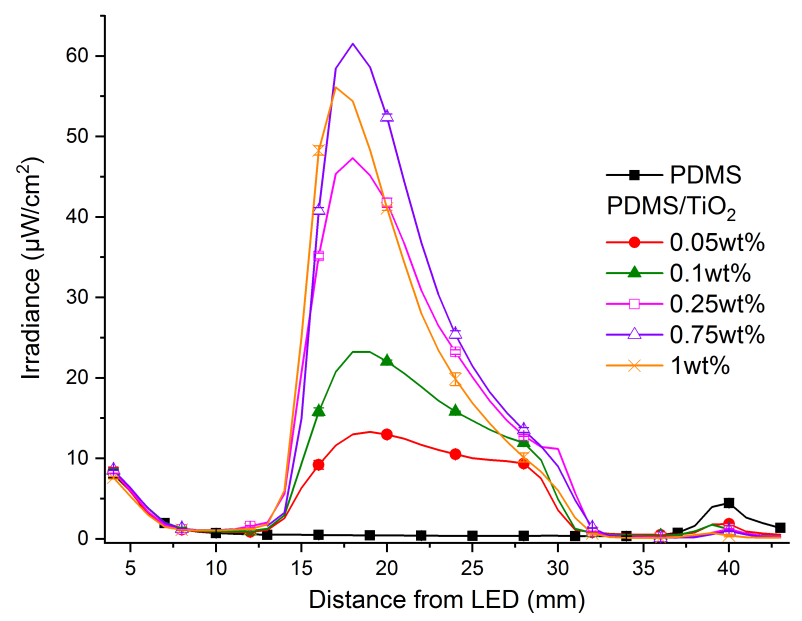

Fig. 3: Irradiance as a function of distance from the LED with different wt $\% \mathrm{TiO}_{2} / \mathrm{PDMS}$ scatter substrates 


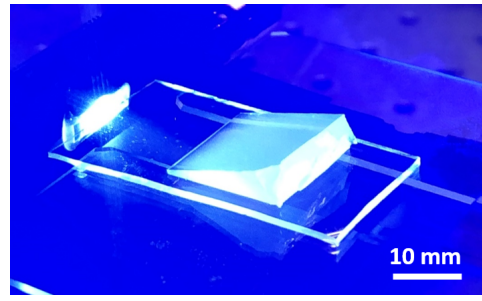

(a)

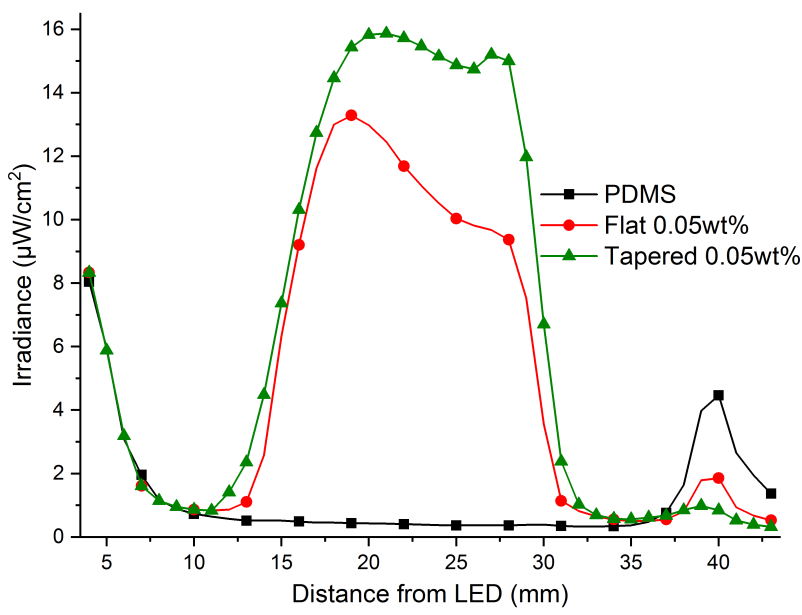

(b)

Fig. 4: Image of the uniform irradiance membrane (a) irradiance as a function of distance from the LED of the flat and tapered $0.05 \mathrm{wt} \%$ $\mathrm{TiO}_{2} / \mathrm{PDMS}$ scatter substrates(b)

centration increases, the maximum irradiance value increases until a maximum irradiance of $61.5 \mu \mathrm{W} / \mathrm{cm}^{2}$ is obtained at $0.75 \mathrm{wt} \%$. At higher concentrations, the $\mathrm{TiO}_{2} / \mathrm{PDMS}$ substrate becomes opaque and less scattered light is extracted. As well as an increased irradiance maximum, higher concentrations of $\mathrm{TiO}_{2}$ have a steeper drop off in irradiance with increasing distance from the LED, with the lower concentrations $(0.05 \mathrm{wt} \%$ and $0.1 \mathrm{wt} \%)$ provide a more uniform irradiance profile but with lower irradiance maximum values of $13.5 \mu \mathrm{W} / \mathrm{cm}^{2}$ and $23 \mu \mathrm{W} / \mathrm{cm}^{2}$ respectively.

\section{B. Uniform light extraction}

Decreasing the $\mathrm{TiO}_{2}$ concentration to a lower value should produce uniform emission based on the trends of Fig. 3, however this would result in lower extracted light and a lower irradiance value. Another method for producing a uniform emission is to utilize a tapered scatter substrate, which gets thicker with increasing distance from the LED therefore increasing the amount of light scattering. Utilizing a low $\mathrm{TiO}_{2}$ concentration of $0.05 \mathrm{wt} \%$, a uniform irradiance of $15 \mu \mathrm{W} / \mathrm{cm}^{2}$ is achieved along a length of $14 \mathrm{~mm}$ with a standard deviation based error of $7 \%$, as shown in Fig. 4. In comparison, the flat $0.05 \mathrm{wt} \%$ scatter substrate produces an average irradiance of $11 \mu \mathrm{W} / \mathrm{cm}^{2}$ with an error of over $15 \%$. As well as providing a uniform irradiance value, the tapered scatter substrate is also more efficient at extracting light, as the irradiance is higher than the flat substrate with the same concentration of $\mathrm{TiO}_{2}$.

\section{Irradiance mapping}

The irradiance results obtained with the spectrometer only account for one spatial direction. Using a CCD imaging system the effect of the scatter substrate can be measured in both the $\mathrm{x}$ and $\mathrm{y}$ directions. Images were taken of the full scatter substrates and the pixel data analyzed to produce a 2-dimensional map of irradiance for each sample. Fig. 5a shows the irradiance map of the $0.05 \mathrm{wt} \% \mathrm{TiO}_{2}$ flat scatter substrate. As can be seen, most of the light is lost closest to the $\mu \mathrm{LED}$, with the distal end of the substrate showing a decreased irradiance, as expected from the previous measurement. Fig. 5b shows the irradiance map of the uniform scatter substrate using $0.05 \mathrm{wt} \% \mathrm{TiO}_{2}$ with a $15^{\circ}$ taper angle. Unlike the flat substrate, the maximum irradiance is broad across the length, as was seen in the irradiance graph (Fig. 4b). The light extraction across the width of the scatter substrates is shown to be uniform, with only the edges showing a decreased irradiance compared to the central regions.

\section{DISCUSSION}

As expected, when a thin PDMS membrane is coupled to the $\mu$ LED device most of the light is guided. Utilizing high refractive index nanoparticles mixed with PDMS as scatter substrates allows for the guided light to be extracted. The amount of light extracted is dependent on the concentration of $\mathrm{TiO}_{2}$ nanoparticles in the PDMS; higher concentrations

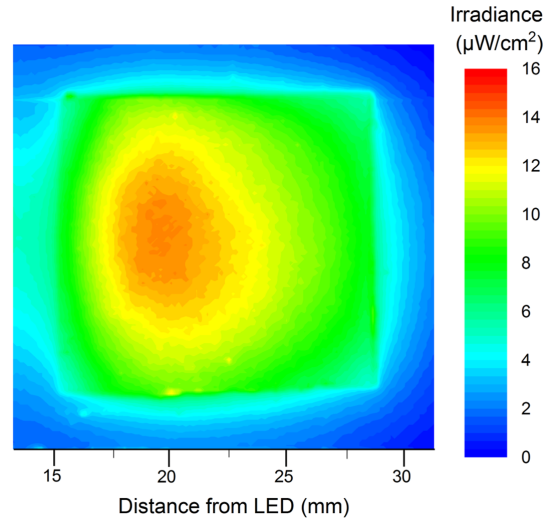

(a)

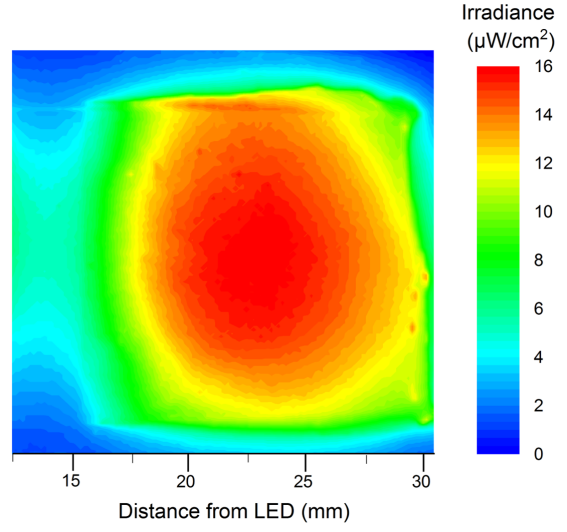

(b)

Fig. 5: 2D irradiance maps for the $0.05 \mathrm{wt} \%$ flat scatter substrate (a) and tapered scatter substrate (b) 
extract more light, until a maximum extraction limit is reached at $0.75 \mathrm{wt} \% \mathrm{TiO}_{2}$. Although efficient at extracting more light, the higher concentration samples produce a highly non-uniform emission profile whilst the lower concentrations extract less light, but have a more uniform profile. A uniform emission of $15 \mu \mathrm{W} / \mathrm{cm}^{2}$ can be obtained using a tapered scatter profile however, this increases the device thickness to a maximum of $5 \mathrm{~mm}$ resulting in decreased wearability. Other methods for obtaining uniform light extraction are being studied, these should allow for a higher irradiance value, whilst maintaining a thinner overall device. Due to the small size of the $\mu$ LEDs, the thickness can be further decreased by coupling to a thinner PDMS membrane. The membrane thickness could be decreased to at least $500 \mu \mathrm{m}$ whilst maintaining a high coupling efficiency. Membranes thinner than this would result in decreased coupling efficiencies and alignment issues [15].

The irradiance values of current phototherapy devices vary based on the wavelength, condition to be treated and where the treatment will be taking place, these values range from sub-mW/cm ${ }^{2}$ to 10 's of $\mathrm{mW} / \mathrm{cm}^{2}$ [7], [8], [10]. Higher irradiance values provide the light dose in a faster time, however high irradiance is also associated with increased side-effects [16]. The wearability of our device should allow for the same treatment dosage to be administered over a longer period of time, resulting in less pain for the patient.

\section{CONCLUSIONS}

We have designed a flexible, conformable device that provides uniform illumination over an area of $2 \mathrm{~cm}^{2}$. Future development of our device will focus on scalability and increased light extraction whilst decreasing the thickness to ensure device is fully wearable.

\section{ACKNOWLEDGMENT}

We are grateful to the UK Engineering and Physical Sciences Research Council, Fraunhofer UK Research Ltd. and the Centre for Doctoral Training in Medical Devices and Health Technologies. This work was supported by EPSRC grant EP/L015595/1.

\section{REFERENCES}

[1] J. Kim, P. Gutruf, A. M. Chiarelli, S. Y. Heo, K. Cho, Z. Xie, A. Banks, S. Han, K.-I. Jang, J. W. Lee, K.-T. Lee, X. Feng, Y. Huang, M. Fabiani, G. Gratton, U. Paik, and J. A. Rogers, "Miniaturized battery-free wireless systems for wearable pulse oximetry," Advanced Functional Materials, vol. 27, no. 1, p. 1604373, 2017.
[2] C. Cochrane, S. R. Mordon, J. C. Lesage, and V. Koncar, "New design of textile light diffusers for photodynamic therapy," Materials Science and Engineering: $C$, vol. 33, no. 3, pp. 1170 - 1175, 2013.

[3] B. M. Quandt, M. S. Pfister, J. F. Lübben, F. Spano, R. M. Rossi, G.-L. Bona, and L. F. Boesel, "POF-yarn weaves: controlling the light outcoupling of wearable phototherapy devices," Biomed. Opt. Express, vol. 8, no. 10, pp. 4316-4330, 2017.

[4] N. B. Meduri, T. Vandergriff, H. Rasmussen, and H. Jacobe, "Phototherapy in the management of atopic dermatitis: a systematic review," Photodermatology, Photoimmunology \& Photomedicine, vol. 23, no. 4, pp. 106-112, 2007.

[5] S. Y. Lee, C. E. You, and M. Y. Park, "Blue and red light combination LED phototherapy for acne vulgaris in patients with skin phototype IV," Lasers in Surgery and Medicine, vol. 39, no. 2, pp. 180-188, 2007.

[6] D. Barolet, "Light-emitting diodes (LEDs) in dermatology," Seminars in cutaneous medicine and surgery, vol. 27, pp. 227-38, 2009.

[7] X. Wen, Y. Li, and M. R. Hamblin, "Photodynamic therapy in dermatology beyond non-melanoma cancer: an update," Photodiagnosis and Photodynamic Therapy, vol. 19, pp. 140 - 152, 2017.

[8] S. H. Ibbotson and J. Ferguson, "Ambulatory photodynamic therapy using low irradiance inorganic light-emitting diodes for the treatment of non-melanoma skin cancer: an open study," Photodermatology, Photoimmunology \& Photomedicine, vol. 28, no. 5, pp. 235-239, 2012.

[9] S. Pfaff, J. Liebmann, M. Born, H. F. Merk, and V. V. Felbert, "Prospective randomized long-term study on the efficacy and safety of UV-free blue light for treating mild psoriasis vulgaris," Dermatology, vol. 231 , p. 24-34, 2015.

[10] H. Moseley, "Light distribution and calibration of commercial PDT LED arrays," Photochem. Photobiol. Sci., vol. 4, pp. 911-914, 2005.

[11] D. Massoubre, E. Xie, B. Guilhabert, J. Herrnsdorf, E. Gu, I. M. Watson, and M. D. Dawson, "Micro-structured light emission from planar InGaN light-emitting diodes," Semiconductor Science and Technology, vol. 29, no. 1, p. 015005, 2013.

[12] R. X. G. Ferreira, E. Xie, J. J. D. McKendry, S. Rajbhandari, H. Chun, G. Faulkner, S. Watson, A. E. Kelly, E. Gu, R. V. Penty, I. H White, D. C. O'Brien, and M. D. Dawson, "High bandwidth GaNbased micro-LEDs for multi-Gb/s visible light communications," IEEE Photonics Technology Letters, vol. 28, no. 19, pp. 2023-2026, 2016.

[13] J.-H. Lee, H.-S. Lee, B.-K. Lee, W.-S. Choi, H.-Y. Choi, and J.B. Yoon, "Design and fabrication of a micropatterned polydimethylsiloxane (PDMS) light-guide plate for sheet-less LCD backlight unit," Journal of the Society for Information Display, vol. 16, no. 2, pp. 329$335,2008$.

[14] M. C. Hudson, "Calculation of the maximum optical coupling efficiency into multimode optical waveguides," Appl. Opt., vol. 13, no. 5, pp. 1029-1033, 1974.

[15] L. Zhu, C. W. Ng, N. Wong, K. K. Y. Wong, P. T. Lai, and H. W Choi, "Pixel-to-pixel fiber-coupled emissive micro-light-emitting diode arrays," IEEE Photonics Journal, vol. 1, no. 1, pp. 1-8, 2009.

[16] J. M. Ang, I. B. Riaz, M. U. Kamal, G. Paragh, and N. C. Zeitouni, "Photodynamic therapy and pain: A systematic review," Photodiagnosis and Photodynamic Therapy, vol. 19, pp. 308 - 344, 2017. 